\title{
OPTIMAL ORDER AND EFFICIENCY FOR ITERATIONS WITH TWO EVALUATIONS*
}

\author{
H. T. KUNG AND J. F. TRAUB†
}

\begin{abstract}
The problem is to calculate a simple zero of a nonlinear function $f$. We consider rational iterations without memory which use two evaluations of $f$ or its derivatives. It is shown that the optimal order is 2 . This settles a conjecture of Kung and Traub that an iteration using $n$ evaluations without memory is of order at most $2^{n-1}$, for the case $n=2$.

Furthermore we show that any rational two-evaluation iteration of optimal order must use either two evaluations of $f$ or one evaluation of $f$ and one of $f^{\prime}$. From this result we completely settle the question of the optimal efficiency, in our efficiency measure, for any two-evaluation iteration without memory. Depending on the relative cost of evaluating $f$ and $f^{\prime}$, the optimal efficiency is achieved by either Newton iteration or the iteration $\psi$ defined by
\end{abstract}

$$
\psi(f)(x)=x-\frac{f^{2}(x)}{f(x+f(x))-f(x)} .
$$

1. Introduction. We deal with optimal iteration for calculating a simple zero of a scalar function $f$ of one variable, which is a prototypical problem of analytic computational complexity (Traub [9]). Early work on this problem appears in Traub [7], [8] while recent results are due to Brent, Winograd and Wolfe [1], Hindmarsh [2], Kung and Traub [3], [4], Rissanen [5] and Wozniakowski [12]. Surveys of recent advances are given by Traub [10], [11]. In this paper, we consider only iterations without memory.

Kung and Traub [4] observe that a reasonable efficiency measure of an iteration $\varphi$ with respect to $f$ should be defined as

$$
e(\varphi, f)=\frac{\log _{2} p(\varphi)}{v(\varphi, f)+a(\varphi)},
$$

where $p(\varphi)$ is the order of convergence of $\varphi, v(\varphi, f)$ is the evaluation cost and $a(\varphi)$ is the combinatory cost. For a given $f$, we are interested in finding an upper bound on $e(\varphi, f)$ and hopefully obtaining an iteration which attains this upper bound. To bound $e(\varphi, f)$ we must know the dependence of $p(\varphi), v(\varphi, f)$, and $a(\varphi)$ on $n$, the number of evaluations.

Let $P_{n}$ denote the maximal order achievable by an iteration using $n$ evaluations. Kung and Traub [3] conjecture that, for iterations without memory,

$$
P_{n} \leqq 2^{n-1}, \quad n=1,2, \cdots .
$$

In this paper, we study rational two-evaluation iterations without memory. We settle the conjecture for $n=2$. We could define and analyze rational oneevaluation iterations without memory and prove the conjecture for $n=1$. Since the proof follows from straightforward modifications of our theorems, we shall

\footnotetext{
* Received by the editors December 11, 1973, and in revised form May 1, 1975.

$\dagger$ Department of Computer Science, Carnegie-Mellon University, Pittsburgh, Pennsylvania 15213. This research was supported in part by the National Science Foundation under Grant GJ32111 and the Office of Naval Research under Contract N0014-67-A-0314-0010, NR044-422.
} 
only indicate these modifications in passing. (This is done after Theorem 4.2.) However, the techniques we use here are suitable for small values of $n$ only. It seems to us that the proof of the conjecture for general $n$ will require the development of new techniques.

Furthermore, we show (Theorem 4.1) that for any rational two-evaluation iteration $\varphi(f)(x)$, one of the two evaluations must be of $f$ at the point $x$. A straightforward modification of this theorem proves that any "locally" convergent iteration requires the evaluation of $f$ at $x$. In Theorem 4.3, we show that the second evaluation of a rational two-evaluation iteration of order $\geqq 2$ must be of either $f$ or $f^{\prime}$ at $x+O(f(x))$.

In this paper, we define cost to be the number of arithmetic operations needed. Let $E_{2}(f)$ denote the optimal efficiency achievable by a rational twoevaluation iteration withcut memory. We show that in our efficiency measure, given by (1.1),

$$
E_{2}(f)=\max \left(\frac{1}{c(f)+c\left(f^{\prime}\right)+2}, \frac{1}{2 c(f)+5}\right)
$$

where $c(f)$ and $c\left(f^{\prime}\right)$ are the costs of evaluating $f$ and $f^{\prime}$, respectively. Depending on the relative cost of evaluating $f$ or $f^{\prime}$, the optimal efficiency $E_{2}(f)$ is achieved by either Newton iteration

$$
\gamma(f)(x)=x-\frac{f(x)}{f^{\prime}(x)}
$$

or the iteration $\psi$,

$$
\psi(f)(x)=x-\frac{f^{2}(x)}{f(x+f(x))-f(x)}
$$

The iteration $\psi$ is derived by Traub $[8, \S 8-4]$. It may also be derived as a special case of Steffensen's iteration [6].

Basic concepts are introduced in $\S 2$. In $\S 3$, we outline the proof of optimal order for $n=2$, with the details given in the following section. Optimal efficiency is studied in the final section.

2. Basic concepts. Let $D=\{f \mid f$ is a real analytic function defined in an open interval $I_{f} \subset R$ (the set of real numbers) which contains a simple zero $\alpha_{f}$ of $f$ and $f^{\prime}$ does not vanish on $\left.I_{f}\right\}$.

Let $\varphi$ be a function which maps every $f \in D$ to $\varphi(f)$ with the following properties:

1. $\varphi(f)$ is a function mapping $I_{\varphi, f} \subset I_{f}$ into $I_{\varphi, f}$ for some open subinterval $I_{\varphi, f}$ containing $\alpha_{f}$

2. $\varphi(f)\left(\alpha_{f}\right)=\alpha_{f}$

3. There exists an open subinterval $I_{\varphi, f}^{0} \subset I_{\varphi, f}$ containing $\alpha_{f}$ such that if $x_{i+1}=\varphi(f)\left(x_{i}\right)$, then $\lim _{i \rightarrow \infty} x_{i}=\alpha_{f}$ whenever $x_{0} \in I_{\varphi, f}^{0}$; 
4. There exist functions $U_{0}^{\prime}, U_{1}, U_{2}$ and nonnegative integers $h, k$, independent of $f$, such that

(i) $U_{0}: R \rightarrow R$ is a rational function,

(ii) $U_{1}: R^{2} \rightarrow R$ is defined formally by

$$
U_{1}(x, y)=\sum_{0}^{l} a_{i}(x) y^{i}
$$

where $a_{i}: R \rightarrow R$ is a rational function,

(iii) $U_{2}: R^{3} \rightarrow R$ is defined formally by

$$
U_{2}(x, y, z)=x+\frac{\sum_{0}^{m} b_{i, j}(x) y^{i} z^{j}}{\sum_{0}^{q} c_{i, j}(x) y^{i} z^{j}},
$$

where $b_{i, j}, c_{i, j}: R \rightarrow R$ are rational functions,

(iv) for all $f \in D$,

$$
\varphi(f)(x)=U_{2}\left(x, f^{(h)}\left(z_{0}\right), f^{(k)}\left(z_{1}\right)\right)
$$

where

$$
\begin{aligned}
& z_{0}=U_{0}(x), \\
& z_{1}=U_{1}\left(x, f^{(h)}\left(z_{0}\right)\right) .
\end{aligned}
$$

If $f^{(h)}\left(z_{0}\right) \neq f^{(k)}\left(z_{1}\right)$ for some $f \in D$ and both $f^{(h)}\left(z_{0}\right)$ and $f^{(k)}\left(z_{1}\right)$ appear formally in $\varphi(f)(x)$, we say $\varphi$ is a rational two-evaluation iteration without memory. Let $\Omega_{2}$ denote the set of all rational two-evaluation iterations without memory.

For $\varphi \in \Omega_{2}, U_{0}$ and $h$ can be chosen such that $U_{0}(x) \equiv x$ and $h=0$ (Theorem 4.1). Hence by (2.3) and (2.4),

$$
\varphi(f)(x)=x+\frac{\sum_{0}^{m} b_{i, j}(x) f^{i}(x)\left(f^{(k)}\left(z_{1}\right)\right)^{j}}{\sum_{0}^{q} c_{i, j}(x) f^{i}(x)\left(f^{(k)}\left(z_{1}\right)\right)^{j}} .
$$

By (2.1) and (2.5),

$$
\begin{gathered}
f^{(k)}\left(z_{1}\right)=f^{(k)}(x)+f^{(k+1)}(x)\left(\sum_{0}^{l} a_{i} f^{i}(x)-x\right)+\frac{1}{2} f^{(k+2)}(x)\left(\sum_{0}^{l} a_{i} f^{i}(x)-x\right)^{2} \\
+\cdots
\end{gathered}
$$

Substituting the right-hand side of (2.7) for $f^{(k)}\left(z_{1}\right)$ in (2.6), we can express $\varphi(f)(x)$ formally in terms of $x, f(x), f^{(k)}(x), f^{(k+1)}(x), \cdots$. Hence we can define functions $\lambda_{i}: R \rightarrow R$ such that

$$
\varphi(f)(x)=\sum_{-\infty}^{\infty} \lambda_{i}(x) f^{i}(x)
$$

where $\lambda_{i}(x)$ depend explicitly on $x, f^{(k)}(x), f^{(k+1)}(x), \cdots$, but not on $f(x)$. It is often desirable to express $\varphi(f)(x)$ by (2.8). We call the right-hand side of (2.8) the canonical form of $\varphi(f)(x)$. 
By the Taylor series expansion of $\varphi(f)(x)$ at $\alpha_{f}$, we can easily show that there exists a nonnegative integer $p(\varphi)$ such that for any $f \in D$,

$$
\lim _{x \rightarrow \alpha_{f}} \frac{\varphi(f)(x)-\alpha_{f}}{\left(x-\alpha_{f}\right)^{p(\varphi)}}=S(\varphi, f)
$$

exists for a constant $S(\varphi, f)$ and $S(\varphi, f) \neq 0$ for at least one $f \in D$. We define $p(\varphi)$ as the order of convergence (order) of $\varphi$.

We assume that one arithmetic operation takes one unit time. Let $c\left(f^{(i)}\right)$ denote the time needed to evaluate $f^{(i)}, i=h, k$. Let $a(\varphi)$ denote the time to compute $\varphi(f)(x)$ from $x$ not counting the time to evaluate $f^{(h)}$ and $f^{(k)}$. We define the efficiency of $\varphi \in \Omega_{2}$ with respect to $f \in D$ as

$$
e(\varphi, f)=\frac{\log _{2} p(\varphi)}{c\left(f^{(h)}\right)+c\left(f^{(k)}\right)+a(\varphi)} .
$$

(See Kung and Traub [4].)

We could easily define iteration without memory and order of convergence in a more general setting, and some of our theorems would still hold. We have chosen here to limit our scope because we wish to focus on optimal efficiency. This is settled by Theorem 5.1, where the hypothesis of rational two-evaluation iteration is crucial. Furthermore, we wish to avoid complicating the proofs. Specifically, Theorem 4.1 holds for analytic iterations satisfying properties 2 and 3 . Theorem 4.2 can be proved for analytic iterations. (See Traub $[8, \S 5.1]$ and Kung and Traub [3, Thm. 6.1].) Furthermore, Theorem 4.4 can be proved for analytic two-evaluation iterations if infinite series are used in (2.1) and (2.2).

3. Outline of the proof of optimal order. Since the proofs of the following section are rather detailed, we summarize the ideas and results here.

In our definition of rational two-evaluation iteration without memory, $\varphi(f)(x)$, we permit any two evaluations of $f$ or its derivatives at any two points. In Theorems 4.1 and 4.3, we cut down the "search space" of evaluations. In Theorems 4.1, we show that one of the evaluations must be of $f$ itself at the point $x$. In Theorem 4.3, we show that the second evaluation of a rational two-evaluation iteration of order $\geqq 2$ must be of either $f$ or $f^{\prime}$ at the point $x+O(f(x))$. Thus the only rational two-evaluation iterations of order $\geqq 2$ are those using either

(i) two evaluations of $f$, or

(ii) one evaluation of $f$ and one of $f^{\prime}$.

In Theorem 4.2, we study the functions $\lambda_{i}(x)$ occurring in the canonical form of the iteration $\varphi$,

$$
\varphi(f)(x)=\sum_{i=-\infty}^{\infty} \lambda_{i}(x) f^{i}(x)
$$

It is easy to check that the iterations $\gamma$ and $\psi$, defined by (1.3) and (1.4) respectively, both have order 2 and canonical form

$$
x-\frac{1}{f^{\prime}(x)} f(x)+O\left(f^{2}(x)\right) .
$$


In Theorem 4.2, we show that any iteration with order $\geqq 2$ has canonical form of the type given in (3.1), and that any iteration of order $\geqq 3$ has canonical form of the type given by

$$
x-\frac{1}{f^{\prime}(x)} f(x)-\frac{f^{\prime \prime}(x)}{2\left(f^{\prime}(x)\right)^{3}} f^{2}(x)+O\left(f^{3}(x)\right) .
$$

(The formulas for $\lambda_{i}(x)$ for iterations of arbitrary order were established by Traub $[8, \S 5.1]$ in a somewhat different setting. See also Kung and Traub [3, Thm. 6.1].)

The main result on optimal order is given in Theorem 4.4 which states that a rational two-evaluation iteration without memory has order at most two. The proof uses a "comparison series" technique first exploited by Traub [7], [8, especially Thms. 5.2, 5.3 and Chap. 9] and also by Kung and Traub [3, Thm. 6.1]. We compare the canonical form of a rational two-evaluation iteration with the canonical form given by (3.2) and show these forms must be different. Hence the order is less than 3 , and since order is an integer in our setting, this implies the order is at most 2 .

\section{Optimal order.}

THEOREM 4.1. If $\varphi \in \Omega_{2}$, then $U_{0}(x) \equiv x$ and $h=0$, or else $U_{1}(x, y) \equiv x$ and $k=0$. Therefore, without loss of generality, we assume that $U_{0}(x) \equiv x$ and $h=0$ in the rest of the paper.

Remark. Although in $\S 2$ it was assumed that $U_{0}$ and $U_{1}$ are rational, the proof of this theorem requires only that one of $U_{0}, U_{1}$ be continuous and the other one be analytic.

Proof. For $\varphi \in \Omega_{2}, f \in D$ and $x \in I_{\varphi, f}$, define

$$
\begin{aligned}
& z_{0}(x)=U_{0}(x), \\
& y_{0}^{h}(x)=f^{(h)}\left(z_{0}(x)\right), \\
& z_{1}(x)=U_{1}\left(x, y_{0}^{h}(x)\right), \\
& y_{1}^{k}(x)=f^{(k)}\left(z_{1}(x)\right) .
\end{aligned}
$$

Then by (2.3),

$$
\varphi(f)(x)=U_{2}\left(x, y_{0}^{h}(x), y_{1}^{k}(x)\right)
$$

Therefore, by (4.2),

$$
\alpha_{f}=\varphi(f)\left(\alpha_{f}\right)=U_{2}\left(\alpha_{f}, y_{0}^{h}\left(\alpha_{f}\right), y_{1}^{k}\left(\alpha_{f}\right)\right) .
$$

We shall first show that either $U_{0}(x) \equiv x$ or $U_{1}(x, y) \equiv x$. Suppose that $U_{0}(x) \not \equiv x$ and $U_{1}(x, y) \not \equiv x$. Assume that $U_{0}$ is continuous and $U_{1}$ is analytic. Since $U_{0}(x) \not \equiv x$, there exists $w_{0} \in R$ such that $U_{0}\left(w_{0}\right) \neq w_{0}$. By the continuity of $U_{0}, U_{0}(w) \neq w$ for all $w \in I_{0}$, for some open interval $I_{0}$ containing $w_{0}$. Choose $f \in D$ such that $\alpha_{f}=w_{0}$ and $z_{1}(x) \not \equiv x$, where $z_{1}(x)$ is defined by (4.1). Since $U_{1}$ and $f$ are analytic, $z_{1}$ is analytic. Hence $\left\{x \mid z_{1}(x)=x\right\}$ has measured zero. We shall show that

$$
\varphi(f)(w)=w
$$

for all $w \in I_{0} \cap I_{\varphi, f}^{0}$ such that $z_{1}(w) \neq w$. By (4.2), we have

$$
\varphi(f)(w)=U_{2}\left(w, y_{0}^{h}(w), y_{1}^{k}(w)\right) .
$$


Since $z_{0}(w)=U_{0}(w) \neq w$ and $z_{1}(w) \neq w$, there exists a polynomial $q$ such that

$$
\begin{aligned}
& q(w)=0, \\
& q^{\prime}(w)=1, \\
& q^{(h)}\left(z_{0}(w)\right)=y_{0}^{h}(w), \\
& q^{(k)}\left(z_{1}(w)\right)=y_{1}^{k}(w) .
\end{aligned}
$$

Obviously, $q \in D$ and $\alpha_{q}=w$. By (4.3) with $f$ replaced by $q$,

$$
w=U_{2}\left(w, y_{0}^{h}(w), y_{1}^{k}(w)\right) .
$$

Equations (4.4) and (4.5) imply that $\varphi(f)(w)=w$. Hence for any $x_{0} \in I_{0} \cap I_{\varphi, f}^{0}$ such that $z_{0}\left(x_{0}\right) \neq x_{0}, \varphi(f)\left(x_{0}\right)=x_{0}$, which does not converge to $\alpha_{f}$. This contradicts property 3 in $\S 2$. Therefore, either $U_{0}(x) \equiv x$ or $U_{1}(x) \equiv x$. This can be proved in the same manner if we assume that $U_{1}$ is continuous and $U_{0}$ is analytic.

To prove the theorem it suffices to show the following:

(i) if $U_{1}(x, y) \not \equiv x$, then $U_{0}(x) \equiv x$ and $h=0$;

(ii) if $U_{1}(x, y) \equiv x$ and $k \neq 0$, then $U_{0}(x) \equiv x$ and $h=0$;

(iii) if $U_{0}(x) \not \equiv x$, then $U_{1}(x, y) \equiv x$ and $k=0$;

(iv) if $U_{0}(x) \equiv x$ and $h \neq 0$, then $U_{1}(x, y) \equiv x$ and $k=0$.

We shall only prove (i), since (ii), (iii), (iv) can be proved similarly. Assume that $U_{1}(x, y) \neq x$. By the first part of the proof, this implies that $U_{0}(x) \equiv x$. Suppose that $h \geqq 1$. Choose $f \in D$ such that $z_{1}(x) \not \equiv x$. Then for any fixed $w \in I_{\varphi, f}^{0}$ such that $z_{1}(w) \neq w$, there exists a polynomial $q$ with the following properties:

$$
\begin{aligned}
& q(w)=0, \\
& q^{(h)}(w)=f^{(h)}(w), \\
& q^{(k)}\left(z_{1}(w)\right)=f^{(k)}\left(z_{1}(w)\right) .
\end{aligned}
$$

Clearly, $q \in D$ and $\alpha_{q}=w$. Therefore we can again show that $\varphi(f)(w)=w$. This is a contradiction.

To simplify notation, in the rest of the paper we shall often write $f^{(i)}, \lambda_{i}, a_{i}, b_{i, j}, c_{i, j}$ for $f^{(i)}(x), \lambda_{i}(x), a_{i}(x), b_{i, j}(x), c_{i, j}(x)$, respectively.

Recall that for $\varphi \in \Omega_{2}$, we can express $\varphi(f)(x)$ by its canonical form, i.e.,

$$
\varphi(f)(x)=\sum_{-\infty}^{\infty} \lambda_{i}(x) f^{i}(x)
$$

THEOREM 4.2.

(i) If $p(\varphi) \geqq 2$, then $\lambda_{i}=0$ for $i<0, \lambda_{0}=x$ and $\lambda_{1}=-1 / f^{\prime}$.

(ii) If $p(\varphi) \geqq 3$, then $\lambda_{i}=0$ for $i<0, \lambda_{0}=x, \lambda_{1}=-1 / f^{\prime}$ and $\lambda_{2}=-f^{\prime \prime} /\left(2 f^{\prime 3}\right)$.

Proof. We shall only prove the second part of the theorem. The first part may be proved analogously.

Define an iteration $\hat{\gamma}$ such that for any $f \in D$,

$$
\hat{\gamma}(f)(x)=x-\frac{f}{f^{\prime}}-\frac{f^{\prime \prime}}{2 f^{\prime 3}} f^{2} .
$$


It is well known that $p(\hat{\gamma})=3$. (For example, see Traub $[8, \S 5.1]$.) Define

$$
T(f)(x)=\frac{\varphi(f)(x)-\hat{\gamma}(f)(x)}{f^{3}} .
$$

Then by (4.6) and (4.7),

$$
T(f)(x)=\sum_{-\infty}^{-1} \lambda_{i} f^{i-3}+\left(\lambda_{0}-x\right) f^{-3}+\left(\lambda_{1}+\frac{1}{f^{\prime}}\right) f^{-2}+\left(\lambda_{2}+\frac{f^{\prime \prime}}{2 f^{\prime 3}}\right) f^{-1}+\sum_{3}^{\infty} \lambda_{i} f^{i-3} .
$$

Suppose that $p(\varphi) \geqq 3$. Then, since $p(\hat{\gamma})=3,(4.8)$ implies that

$$
\lim _{x \rightarrow \alpha_{f}}|T(f)(x)|<\infty
$$

for all $f \in D$. Hence it follows from (4.9) that

$$
\lambda_{i}=0 \quad \text { for } i<0, \quad \lambda_{0}=x, \quad \lambda_{1}=-\frac{1}{f^{\prime}} \quad \text { and } \quad \lambda_{2}=-\frac{f^{\prime \prime}}{2 f^{\prime 3}} .
$$

From Theorems 4.1 and 4.2 , we can immediately prove the conjecture (1.2) for $n=1$ as follows: Let $\varphi(f)(x)$ be a rational one-evaluation iteration without memory. Then by a straightforward modification of Theorem 4.1 the evaluation must be of $f$ at $x$. Hence

$$
\varphi(f)(x)=H(x, f(x))
$$

for some rational function $H: R^{2} \rightarrow R$. It follows that the canonical form of $\varphi$ cannot be given by

$$
x-\frac{1}{f^{\prime}(x)} f(x)+O\left(f^{2}(x)\right)
$$

Therefore, by Theorem 4.2, $p(\varphi)<2$. Since $p(\varphi)$ is an integer, we have $p(\varphi) \leqq 1$. This proves the conjecture (1.2) for $n=1$.

THEOREM 4.3. Let $\varphi \in \Omega_{2}$ and $p(\varphi) \geqq 2$. Then

(i) $k=0$ or 1 ,

(ii) $a_{0}$ is the identity function.

Proof. (i) Suppose that $k \geqq 2$. Then by (2.7) and (2.8) it is clear that $\lambda_{1}(x)$ does not depend on $f^{\prime}(x)$ explicitly. Hence by Theorem 4.2, $p(\varphi)<2$. Thus we have shown the first part of the theorem.

(ii) Assume that $a_{0}(x) \not \equiv x$. Then the set $\left\{x \mid a_{0}(x)=x\right\}$ has measure zero. Note that

$$
f^{(k)}\left(z_{1}\right)=f^{(k)}\left(a_{0}(x)\right)+f^{(k+1)}\left(a_{0}(x)\right)\left(a_{1}(x) f(x)+a_{2}(x) f^{2}(x)+\cdots\right)+\cdots
$$

Suppose that $p(\varphi) \geqq 2$.

Case 1. $(k=0)$. Since $\varphi(f)\left(\alpha_{f}\right)=\alpha_{f}$ and $f\left(\alpha_{f}\right)=0$, by (2.6) and (4.10) we have

$$
\alpha_{f}=\alpha_{f}+\frac{\sum_{0}^{m} b_{0, j}\left(\alpha_{f}\right) f\left(a_{0}\left(\alpha_{f}\right)\right)^{j}}{\sum_{0}^{q} c_{0, j}\left(\alpha_{f}\right) f\left(a_{0}\left(\alpha_{f}\right)\right)^{j}} .
$$


Therefore

$$
\sum_{0}^{m} b_{0, j}\left(\alpha_{f}\right) f\left(a_{0}\left(\alpha_{f}\right)\right)^{j}=0
$$

for all $f \in D$. This implies that

$$
b_{0, j}(x) \equiv 0, \quad j=0, \cdots, m .
$$

Let $r$ be the largest integer such that

$$
b_{i, j}(x) \equiv 0, \quad j=0, \cdots, m, \quad i=0,1, \cdots, r .
$$

Then by (4.11), $r \geqq 0$. Clearly, $r<m$. Note that

$$
\sum_{0}^{m} b_{i, j}(x) f^{i}(x) f^{j}\left(z_{1}\right)=f^{r+1}(x) B(f)(x),
$$

where

$$
B(f)(x)=\sum_{\substack{0 \leqq j \leqq m \\ 0 \leqq i \leqq m-r-1}} b_{i+r+1, j}(x) f^{i}(x) f^{j}\left(z_{1}\right) .
$$

By Theorem 4.2, it is clear that

$$
\varphi(f)^{\prime}\left(\alpha_{f}\right)=0 .
$$

This implies that

$$
1+\frac{(r+1) f^{r}\left(\alpha_{f}\right) f^{\prime}\left(\alpha_{f}\right) B(f)\left(\alpha_{f}\right)}{\sum_{0}^{q} c_{0, j}\left(\alpha_{f}\right) f\left(a_{0}\left(\alpha_{f}\right)\right)^{j}}=0 .
$$

Hence $r$ must be equal to 0 . Note that

$$
B(f)\left(\alpha_{f}\right)=\sum_{0 \leqq j \leqq m} b_{1, j}\left(\alpha_{f}\right) f\left(a_{0}\left(\alpha_{f}\right)\right)^{j} .
$$

Therefore (4.12) is reduced to

$$
\sum_{0 \leqq j \leqq m} b_{1, j}\left(\alpha_{f}\right) f^{\prime}\left(\alpha_{f}\right) f\left(a_{0}\left(\alpha_{f}\right)\right)^{j}+\sum_{0 \leqq j \leqq q} c_{0, j}\left(\alpha_{f}\right) f\left(a_{0}\left(\alpha_{f}\right)\right)^{j}=0
$$

for all $f \in D$. Since for any real number $s$ such that $s \neq a_{0}(s)$ and any real numbers $u$ $(\neq 0), v$ there exists $f \in D$ such that

$$
\begin{aligned}
& f(s)=0, \quad \text { i.e., } \alpha_{f}=s, \\
& f^{\prime}(s)=u, \\
& f\left(a_{0}(s)\right)=v,
\end{aligned}
$$

then by (4.13),

$$
\sum_{0 \leqq j \leqq m} b_{1, j}(s) u v^{j}+\sum_{0 \leqq j \leqq q} c_{0, j}(s) v^{j}=0
$$


holds for any $s, u, v$ such that $u \neq 0$ and $s \neq a_{0}(s)$. Therefore

$$
b_{1, j}(x) \equiv 0, \quad j=0, \cdots, m .
$$

This contradicts the definition of $r$.

Case 2. $(k=1)$. In this case, we substitute

$$
f^{\prime}\left(a_{0}(x)\right)+f^{\prime \prime}\left(a_{0}(x)\right)\left(a_{1}(x) f(x)+a_{2}(x) f(x)^{2}+\cdots\right) \cdots
$$

for $f^{\prime}\left(z_{1}\right)$. Then following the same procedure used in Case 1 , we obtain

$$
\sum_{0 \leqq j \leqq m} b_{1, j}\left(\alpha_{f}\right) f^{\prime}\left(\alpha_{f}\right) f^{\prime}\left(a_{0}\left(\alpha_{f}\right)\right)^{j}+\sum_{0 \leqq j \leqq q} c_{0, j}\left(\alpha_{f}\right) f^{\prime}\left(a_{0}\left(\alpha_{f}\right)\right)^{j}=0
$$

rather than (4.13) for all $f \in D$. By the same reasoning as used before, we get a contradiction.

The main result on optimal order is given as follows.

THEOREM 4.4. If $\varphi$ is a rational two-evaluation iteration without memory, then $p(\varphi) \leqq 2$.

Proof. Suppose that $p(\varphi) \geqq 3$. By Theorem $4.3, k=0$ or 1 and $z_{1}=x+a_{1} f+a_{2} f^{2}+\cdots$. Hence

$$
\begin{gathered}
f\left(z_{1}\right)=\left(1+a_{1} f^{\prime}\right) f+\left(a_{2} f^{\prime}+\left(a_{1}^{2} / 2\right) f^{\prime \prime}\right) f^{2}+\cdots \\
f^{\prime}\left(z_{1}\right)=f^{\prime}+\left(a_{1} f+a_{2} f^{2}+\cdots\right) f^{\prime \prime}+\left(a_{1} f+a_{2} f^{2}+\cdots\right)^{2}\left(f^{\prime \prime \prime} / 2\right)+\cdots \\
=f^{\prime}+a_{1} f^{\prime \prime} f+\left(a_{2} f^{\prime \prime}+\left(a_{1} / 2\right) f^{\prime \prime \prime}\right) f^{2}+\cdots
\end{gathered}
$$

Hence if we substitute the right-hand sides of (4.15) and (4.16) for $f\left(z_{1}\right)$ and $f^{\prime}\left(z_{1}\right)$, respectively, then we can define $\nu_{i}$ and $\mu_{i}$ as follows:

$$
\sum_{0}^{\infty} \nu_{i} f^{i}= \begin{cases}\sum_{0}^{m} b_{i, j} f^{i} f\left(z_{1}\right)^{j} & \text { if } k=0, \\ \sum_{0}^{m} b_{i, j} f^{i} f^{\prime}\left(z_{1}\right)^{j} & \text { if } k=1,\end{cases}
$$

and

$$
\sum_{0}^{\infty} \mu_{i} f^{i}= \begin{cases}\sum_{0}^{q} c_{i, j} f^{i} f\left(z_{1}\right)^{j} & \text { if } k=0 \\ \sum_{0}^{q} c_{i, j} f^{i} f^{\prime}\left(z_{1}\right)^{j} & \text { if } k=1\end{cases}
$$

Then by (2.2),

$$
\varphi(f)(x)=x+\frac{\sum_{0}^{\infty} \nu_{i} f^{i}}{\sum_{0}^{\infty} \mu_{i} f^{i}}
$$


Hence by Theorem 4.2,

$$
\frac{\sum_{0}^{\infty} \nu_{i} f^{i}}{\sum_{0}^{\infty} \mu_{i} f^{i}}=-\frac{1}{f^{\prime}} f-\frac{f^{\prime \prime}}{2 f^{\prime 3}} f^{2}+O\left(f^{3}\right) .
$$

Suppose that $\mu_{n} \neq 0$ and $\mu_{i}=0$ for $i<n$. Then by (4.17),

$$
\begin{gathered}
\nu_{i}=0 \quad \text { for } i<n+1, \\
f^{\prime} \nu_{n+1}=-\mu_{n}, \\
2 f^{\prime 3} \nu_{n+2}=-\mu_{n} f^{\prime \prime}-2 f^{\prime 2} \mu_{n+1} .
\end{gathered}
$$

Case 1. $\left(k=0\right.$ and $\left.a_{1} \neq 0\right)$. It is easy to check that

$$
\begin{aligned}
\mu_{0}= & c_{0,0}, \\
\mu_{1}= & c_{1,0}+c_{0,1}\left(1+a_{1} f^{\prime}\right), \\
\mu_{2}= & c_{2,0}+c_{1,1}\left(1+a_{1} f^{\prime}\right)+c_{0,2}\left(1+a_{1} f^{\prime}\right)^{2}+c_{0,1}\left(a_{2} f^{\prime}+\left(a_{1}^{2} / 2\right) f^{\prime \prime}\right), \\
\mu_{3}= & c_{3,0}+c_{2,1}\left(1+a_{1} f^{\prime}\right)+c_{1,2}\left(1+a_{1} f^{\prime}\right)^{2}+c_{0,3}\left(1+a_{1} f^{\prime}\right)^{3} \\
& +c_{1,1}\left(a_{2} f^{\prime}+\left(a_{1}^{2} / 2\right) f^{\prime \prime}\right)+2 c_{0,2}\left(1+a_{1} f^{\prime}\right)\left(a_{2} f^{\prime}+\left(a_{1}^{2} / 2\right) f^{\prime \prime}\right) \\
& +c_{0,1}\left(a_{3} f^{\prime}+a_{1} a_{2} f^{\prime \prime}+\left(a_{1}^{3} / 6\right) f^{\prime \prime \prime}\right) .
\end{aligned}
$$

Since $\mu_{n} \neq 0$ and $\mu_{i}=0$ for $i<n$, by (4.21) one can easily see that

$$
c_{i, j}=0 \quad \text { whenever } i+j<n .
$$

Hence

$$
\begin{aligned}
\mu_{n}= & c_{n, 0}+c_{n-1,1}\left(1+a_{1} f^{\prime}\right)+\cdots+c_{0, n}\left(1+a_{1} f^{\prime}\right)^{n} \\
\mu_{n+1}= & c_{n+1,0}+c_{n, 1}\left(1+a_{1} f^{\prime}\right)+\cdots+c_{0, n+1}\left(1+a_{1} f^{\prime}\right)^{n+1} \\
& +c_{n-1,1}\left(a_{2} f^{\prime}+\left(a_{1}^{2} / 2\right) f^{\prime \prime}\right)+2 c_{n-2,2}\left(1+a_{1} f^{\prime}\right)\left(a_{2} f^{\prime}+\left(a_{1}^{2} / 2\right) f^{\prime \prime}\right) \\
& +\cdots+n c_{0, n}\left(1+a_{1} f^{\prime}\right)^{n-1}\left(a_{2} f^{\prime}+\left(a_{1}^{2} / 2\right) f^{\prime \prime}\right) .
\end{aligned}
$$

Similarly, by (4.18), we also have

$$
\begin{aligned}
\nu_{n+1}= & b_{n+1,0}+b_{n, 1}\left(1+a_{1} f^{\prime}\right)+\cdots+b_{0, n+1}\left(1+a_{1} f^{\prime}\right)^{n+1} \\
\nu_{n+2}= & b_{n+2,0}+b_{n+1,1}\left(1+a_{1} f^{\prime}\right)+\cdots+b_{0, n+2}\left(1+a_{1} f^{\prime}\right)^{n+2} \\
& +b_{n, 1}\left(a_{2} f^{\prime}+\left(a_{1}^{2} / 2\right) f^{\prime \prime}\right)+2 b_{n-1,2}\left(1+a_{1} f^{\prime}\right)\left(a_{2} f^{\prime}+\left(a_{1}^{2} / 2\right) f^{\prime \prime}\right) \\
& +\cdots+(n+1) b_{0, n+1}\left(1+a_{1} f^{\prime}\right)^{n}\left(a_{2} f^{\prime}+\left(a_{1}^{2} / 2\right) f^{\prime \prime}\right) .
\end{aligned}
$$

From (4.19),

$$
\begin{aligned}
b_{n+1,0} f^{\prime} & +b_{n, 1}\left(1+a_{1} f^{\prime}\right) f^{\prime}+\cdots+b_{2, n-1}\left(1+a_{1} f^{\prime}\right)^{n-1} f^{\prime} \\
& +b_{1, n}\left(1+a_{1} f^{\prime}\right)^{n} f^{\prime}+b_{0, n+1}\left(1+a_{1} f^{\prime}\right)^{n+1} f^{\prime} \\
= & -\left[c_{n, 0}+c_{n-1,1}\left(1+a_{1} f^{\prime}\right)+\cdots+c_{0, n}\left(1+a_{1} f^{\prime}\right)^{n}\right]
\end{aligned}
$$


Comparing the coefficients of $f^{\prime n+2}, f^{\prime n+1}, f^{\prime n}$ in (4.23), we get

$$
\begin{aligned}
& b_{0, n+1}=b_{1, n}=0, \\
& b_{2, n-1}=-a_{1} c_{0, n} .
\end{aligned}
$$

Comparing the coefficients of $f^{\prime \prime}$ in (4.20), we get

$$
\begin{aligned}
-\left[b_{n, 1}\right. & \left.a_{1}^{2} f^{\prime 3}+2 b_{n-1,2}\left(1+a_{1} f^{\prime}\right) a_{1}^{2} f^{\prime 3}+\cdots+(n-1) b_{2, n-1}\left(1+a_{1} f^{\prime}\right)^{n-2} a_{1}^{2} f^{\prime 3}\right] \\
= & c_{n, 0}+c_{n-1,1}\left(1+a_{1} f^{\prime}\right)+\cdots+c_{0, n}\left(1+a_{1} f^{\prime}\right)^{n}+c_{n-1,1} a_{1}^{2} f^{\prime 2} \\
& +2 c_{n-2,2}\left(1+a_{1} f^{\prime}\right) a_{1}^{2} f^{\prime 2} \\
& +\cdots+n c_{0, n}\left(1+a_{1} f^{\prime}\right)^{n-1} a_{1}^{2} f^{\prime 2} .
\end{aligned}
$$

Comparing the coefficients of $f^{\prime n+1}$ in (4.26), we get

$$
(n-1) b_{2, n-1}=-n c_{0, n} a_{1} .
$$

Equations (4.25) and (4.27) imply that

$$
b_{2, n-1}=c_{0, n}=0 .
$$

Equations (4.23) and (4.26) are reduced to

$$
\begin{aligned}
& b_{n+1,0} f^{\prime}+b_{n, 1}\left(1+a_{1} f^{\prime}\right) f^{\prime}+\cdots+b_{3, n-2}\left(1+a_{1} f^{\prime}\right)^{n-2} f^{\prime} \\
& =-\left[c_{n, 0}+c_{n-1,1}\left(1+a_{1} f^{\prime}\right)+\cdots+c_{1, n-1}\left(1+a_{1} f^{\prime}\right)^{n-1}\right],
\end{aligned}
$$

and

$$
\begin{aligned}
& \quad-\left[b_{n, 1} a_{1}^{2} f^{\prime 3}+\cdots+(n-2) b_{3, n-2}\left(1+a_{1} f^{\prime}\right)^{n-3} a_{1}^{2} f^{\prime 3}\right] \\
& \stackrel{(4.29)}{=} c_{n, 0}+\cdots+c_{1, n-1}\left(1+a_{1} f^{\prime}\right)^{n-1}+c_{n-1,1} a_{1}^{2} f^{\prime 2}+\cdots+(n-1) c_{1, n-1}\left(1+a_{1} f^{\prime}\right)^{n-2} a_{1}^{2} f^{\prime 2} .
\end{aligned}
$$

Comparing the coefficients of $f^{\prime n-1}$ and $f^{\prime n}$ in (4.28) and (4.29), respectively, we get

$$
\begin{aligned}
b_{3, n-2} & =-a_{1} c_{1, n-1}, \\
(n-2) b_{3, n-2} & =-(n-1) c_{1, n-1} a_{1} .
\end{aligned}
$$

Equations (4.30) and (4.31) imply that

$$
b_{3, n-2}=c_{1, n-1}=0 \text {. }
$$

By induction we can show that

$$
c_{j, n-j}=0 \quad \text { for } j=0,1, \cdots, n .
$$

Therefore, $\mu_{n}=0$. This is a contradiction.

Case 2. $\left(k=0\right.$ and $\left.a_{1}=0\right)$. Suppose that $a_{i}=0$ for all $i>0$. Then $\varphi$ is a rational one-evaluation iteration. Hence $p(\varphi) \leqq 1<2$ and the theorem is proved. Therefore, here we consider the case that $a_{i} \neq 0$ for some $i \geqq 2$. In the following we shall assume that $a_{2} \neq 0$. (The argument used below can be immediately generalized to the case when the smallest value $i$ such that $a_{i} \neq 0$ is not 2.)

Since $z_{1}=x+a_{2} f^{2}+a_{3} f^{3}+\cdots$,

$$
f\left(z_{1}\right)=f+\left(a_{2} f^{2}+a_{3} f^{3}+\cdots\right) f^{\prime}+\left(a_{2} f^{2}+a_{3} f^{3}+\cdots\right)^{2} \cdot \frac{f^{\prime \prime}}{2}+\cdots .
$$


To simplify notation, we define $A_{1}, A_{2}, \cdots$ by

$$
f\left(z_{1}\right)=A_{1} f+A_{2} f^{2}+A_{3} f^{3}+A_{4} f^{4}+\cdots .
$$

Then $A_{1}=1, A_{2}=a_{2} f^{\prime}, A_{3}=a_{3} f^{\prime}, A_{4}=\left(a_{4} f^{\prime}+\left(a_{2}^{2} / 2\right) f^{\prime \prime}\right)$, etc. Note that $f^{\prime \prime}$ does not appear in $A_{1}, A_{2}$ and $A_{3}$.

It is easy to check that

$$
\begin{aligned}
\mu_{0}= & c_{0,0}, \\
\mu_{1}= & c_{1,0}+c_{0,1} A_{1}, \\
\mu_{2}= & c_{2,0}+c_{1,1} A_{1}+c_{0,2} A_{1}^{2}+c_{0,1} A_{2}, \\
\mu_{3}= & c_{3,0}+c_{2,1} A_{1}+c_{1,2} A_{1}^{2}+c_{0,3} A_{1}^{3}+\left(c_{1,1}+2 c_{0,2} A_{1}\right) A_{2}+c_{0,1} A_{3}, \\
\mu_{4}= & c_{4,0}+c_{3,1} A_{1}+c_{2,2} A_{1}^{2}+c_{1,3} A_{1}^{3}+c_{0,4} A_{1}^{4}+\left(c_{2,1}+2 c_{1,2} A_{1}\right. \\
& \left.+3 c_{0,3} A_{1}^{2}\right) A_{2}+c_{0,2} A_{2}^{2}+\left(c_{1,1}+2 c_{0,2} A_{1}\right) A_{3}+c_{0,1} A_{4}, \\
\mu_{5}= & c_{5,0}+c_{4,1} A_{1}+c_{3,2} A_{1}^{2}+c_{2,3} A_{1}^{3}+c_{1,4} A_{1}^{4}+c_{0,5} A_{1}^{5}+\left(c_{3,1}\right. \\
& \left.+2 c_{2,2} A_{1}+3 c_{1,3} A_{1}^{2}+4 c_{0,4} A_{1}^{3}\right) A_{2}+\left(c_{1,2}+3 c_{0,3} A_{1}\right) A_{2}^{2}+\left(c_{2,1}+2 c_{1,2} A_{1}\right. \\
& \left.+3 c_{0,3} A_{1}^{2}\right) A_{3}+\left(c_{1,1}+2 c_{0,2} A_{1}\right) A_{4}+2 c_{0,2} A_{2} A_{3}+c_{0,1} A_{5},
\end{aligned}
$$

Suppose that $\mu_{i}=0$ for $i<4$. Then since $a_{2} \neq 0$, we have

$$
c_{0,1}=c_{1,1}+2 c_{0,1} A_{1}=0 \text {. }
$$

Hence $\mu_{4}$ does not depend on $A_{i}$ for $i \geqq 3$ and $\mu_{5}$ does not depend on $A_{i}$ for $i \geqq 4$. We shall first prove by induction that, in general, if $\mu_{i}=0$ for $i<n$, then

(i) $\mu_{n}$ does not depend on $A_{i}$ for $i \geqq 3$,

(ii) $\mu_{n+1}$ does not depend on $A_{i}$ for $i \geqq 4$.

Assuming the assertion is true for $n \leqq r$, we shall verify it for $n=r+1$. Suppose that $\mu_{i}=0$ for $i<r+1$. Then, of course, $\mu_{i}=0$ for $i<r$. Hence by the induction hypotheses,

$$
\begin{gathered}
\mu_{r} \quad \text { does not depend on } A_{i} \text { for } i \geqq 3, \\
\mu_{r+1} \quad \text { does not depend on } A_{i} \text { for } i \geqq 4 .
\end{gathered}
$$

We now prove (i) for $n=r+1$. By (4.33), a term in $\mu_{r+1}$ is of the form $A_{1}^{e_{1}} A_{2}^{e_{2}} A_{3}^{e_{3}}$, where

$$
e_{1}+2 e_{2}+3 e_{3} \leqq r+1
$$

It is easy to check that its coefficient is

$$
S\left(e_{1}, e_{2}, e_{3}\right)=\frac{\left(e_{1}+e_{2}+e_{3}\right) !}{e_{1} ! e_{2} ! e_{3} !} c_{(r+1)-\left(e_{1}+2 e_{2}+3 e_{3}\right), e_{1}+e_{2}+e_{3}} .
$$

However, since $A_{1}=1, A_{1}^{e_{1}} A_{2}^{e_{2}} A_{3}^{e_{3}}=A_{2}^{e_{2}} A_{3}^{e_{3}}$. After replacing $A_{1}$ by 1 in $\mu_{r+1}$, we obtain the coefficient of $a_{2}^{e_{2}} A_{3}^{e_{2}}$ in $\mu_{r+1}$, which is

$$
\sum_{e_{1}} S\left(e_{1}, e_{2}, e_{3}\right)
$$


where the summation takes all values of $e_{1}$ satisfying (4.34). Similarly, the coefficient of $A_{2}^{e_{2}+e_{3}}$ in $\mu_{r+1-e_{3}}$ is

$$
\sum_{e_{1}} T\left(e_{1}, e_{2}, e_{3}\right)
$$

where

$$
T\left(e_{1}, e_{2}, e_{3}\right)=\frac{\left(e_{1}+e_{2}+e_{3}\right) !}{e_{1} !\left(e_{2}+e_{3}\right) !} c_{\left(r+1-e_{3}\right)-\left(e_{1}+2 e_{2}+2 e_{3}\right), e_{1}+e_{2}+e_{3}},
$$

and $e_{1}$ satisfies (4.34). Clearly, we have

$$
\sum_{e_{1}} S\left(e_{1}, e_{2}, e_{3}\right)=\frac{\left(e_{2}+e_{3}\right) !}{e_{2} ! e_{3} !} \sum_{e_{1}} T\left(e_{1}, e_{2}, e_{3}\right) .
$$

Now suppose $e_{3} \geqq 1$. Then $r+1-e_{3} \leqq r$. Hence $\mu_{r+1-e_{3}}=0$ and $\mu_{r+1-e_{3}}$ does not depend on $A_{i}$ for $i \geqq 3$. Therefore, the coefficient of $A_{2}^{e_{2}+e_{3}}$ in $\mu_{r+1-e_{3}}$, $\sum_{e_{1}} T\left(e_{1}, e_{2}, e_{3}\right)$, must be zero. By (4.35), the coefficient of $A_{2}^{e_{2}} A_{3}^{e_{3}}$ in $\mu_{r+1}$ is zero. We have shown that (i) holds for $n=r+1$. The proof of (ii) for $n=r+1$ is similar. Let the coefficient of $A_{2}^{e_{2}} A_{3}^{e_{3}} A_{4}^{e_{4}} \cdots A_{t}^{e_{t}}$ in $\mu_{r+2}$ be $\sum_{e_{1}} S\left(e_{1}, \cdots, e_{t}\right)$ and that of $A_{2}^{e_{3}+e_{4}+\cdots+e_{t}} A_{3}^{e_{3}}$ in $\mu_{r+2-\left(2 e_{4}+3 e_{5}+\cdots+(t-2) e_{t}\right)}$ be $\sum_{e_{1}} T\left(e_{1}, \cdots, e_{t}\right)$, where $t \geqq 4$ and

$$
\sum_{1}^{n} i e_{i} \leqq r+2 \text {. }
$$

Then it can be shown that

$$
\sum_{e_{1}} S\left(e_{1}, \cdots, e_{t}\right)=\frac{\left(e_{2}+e_{4}+\cdots+e_{t}\right) !}{e_{2} ! e_{4} ! \cdots e_{t} !} \sum_{e_{1}} T\left(e_{1}, \cdots, e_{t}\right) .
$$

Suppose that $e_{i} \geqq 1$ for some $i \geqq 4$. Then

$$
r+2-\left(2 e_{4}+3 e_{5}+\cdots+(t-2) e_{t}\right) \leqq r .
$$

Hence $\mu_{r+2-\left(2 e_{4} \cdots\right)}=0$ and $\mu_{r+2-\left(2 e_{4}+\cdots\right)}$ does not depend on $A_{3}$. Therefore $e_{3}=0$ and $\sum_{e_{1}} S\left(e_{1}, \cdots, e_{t}\right)=0$. Hence we have shown that (ii) holds for $n=r+1$.

Since $\mu_{i}=0$ for $i<n$, by (4.17), $\nu_{i}=0$ for $i<n+1$. Hence we can similarly show that $\nu_{n+2}$ does not depend on $A_{i}$ for $i \geqq 4$. Since $f^{\prime \prime}$ does not appear in $A_{1}, A_{2}$ and $A_{3}$, we have shown that $f^{\prime \prime}$ does not appear in $\mu_{n}, \mu_{n+1}$ and $\nu_{n+2}$. But by (4.20),

$$
\mu_{n} f^{\prime \prime}=-2 f^{\prime} \nu_{n+2}-2 f^{\prime 2} \mu_{n+1}
$$

Therefore, $\mu_{n}=0$. This is a contradiction.

Case 3. $(k=1)$. In this case, it is easy to check that

$$
\begin{aligned}
\mu_{0}= & c_{0,0}+c_{0,1} f^{\prime}+c_{0,2} f^{\prime 2}+\cdots, \\
\mu_{1}= & c_{1,0}+c_{1,1} f^{\prime}+c_{1,2} f^{\prime 2}+\cdots+c_{0,1} a_{1} f^{\prime \prime}+2 c_{0,2} a_{1} f^{\prime} f^{\prime \prime}+3 c_{0,3} a_{1} f^{\prime 2} f^{\prime \prime}+\cdots \\
= & \left(c_{1,0}+c_{1,1} f^{\prime}+c_{1,2} f^{\prime 2}+\cdots\right)+a_{1} f^{\prime \prime}\left(c_{0,1}+2 c_{0,2} f^{\prime}+3 c_{0,3} f^{\prime 2}+\cdots\right), \\
\mu_{2}= & \left(c_{2,0}+c_{2,1} f^{\prime}+c_{2,2} f^{\prime 2}+\cdots\right)+a_{1} f^{\prime \prime}\left(c_{1,1}+2 c_{1,2} f^{\prime}+3 c_{1,3} f^{\prime 2}+\cdots\right) \\
& +\left(a_{2} f^{\prime \prime}+\left(a_{1} / 2\right) f^{\prime \prime \prime}\right)\left(c_{0,1}+2 c_{0,2} f^{\prime}+3 c_{0,3} f^{\prime 2}+\cdots\right),
\end{aligned}
$$


Since $\mu_{n} \neq 0$ and $\mu_{i}=0$ for $i<n$, by (4.37) one can easily see that

$$
c_{i, j}=0 \text { for } i<n, \quad j=0,1, \cdots .
$$

Hence

$$
\begin{aligned}
& \mu_{n}=c_{n, 0}+c_{n, 1} f^{\prime}+c_{n, 2} f^{\prime 2}+\cdots, \\
& \mu_{n+1}=\left(c_{n+1,0}+c_{n+1,1} f^{\prime}+\cdots\right)+a_{1} f^{\prime \prime}\left(c_{n, 1}+2 c_{n, 2} f^{\prime}+3 c_{n, 3} f^{\prime 2}+\cdots\right) .
\end{aligned}
$$

Similarly, by (4.18), we also have

$$
\begin{aligned}
& \nu_{n+1}=b_{n+1,0}+b_{n+1,1} f^{\prime}+b_{n+1,2} f^{\prime 2}+\cdots \\
& \nu_{n+2}=\left(b_{n+2,0}+b_{n+2,1} f^{\prime}+\cdots\right)+a_{1} f^{\prime \prime}\left(b_{n+1,1}+2 b_{n+1,2} f^{\prime}+3 b_{n+1,3} f^{\prime 2}+\cdots\right) .
\end{aligned}
$$

Comparing the coefficients of $f^{\prime i}, i=0,1, \cdots$ in (4.19), we get

$$
\begin{aligned}
& c_{n, 0}=0, \\
& c_{n, j}=b_{n+1, j-1}, \\
& j=1,2, \cdots .
\end{aligned}
$$

Next, comparing the coefficients of $f^{\prime \prime}$ in (4.20), we get

$$
\begin{aligned}
& 2 a_{1} f^{\prime 3}\left(b_{n+1,1}+2 b_{n+1,2} f^{\prime}+3 b_{n+1,3} f^{\prime 2}+\cdots\right) \\
& \quad=-\left(c_{n, 0}+c_{n, 1} f^{\prime}+c_{n, 2} f^{\prime 2}+\cdots\right)-2 a_{1} f^{\prime 2}\left(c_{n, 1}+2 c_{n, 2} f^{\prime}+\cdots\right) .
\end{aligned}
$$

Hence, comparing coefficients of $f^{\prime i}, i=0,1, \cdots$, in (4.39), we get

$$
\begin{aligned}
& c_{n, 0}=c_{n, 1}=0, \\
& -c_{n, j+1}-2 j a_{1} c_{n, j}=2(j-1) a_{1} b_{n+1, j-1}, \quad j=1,2, \cdots .
\end{aligned}
$$

From (4.38) and (4.40) it is trivial to see that

$$
c_{n, j}=0 \quad \text { for } j=0,1,2, \cdots .
$$

Therefore, $\mu_{n}=0$. This is a contradiction.

\section{Optimal efficiency.}

LEMMA 5.1. If $\varphi \in \Omega_{2}$ and $\varphi$ uses evaluations of $f$ and $f^{\prime}$, then $a(\varphi) \geqq 2$.

Proof. It suffices to show that $\varphi(f)(x)$ depends explicitly on $x, f(x)$ and $f^{\prime}\left(z_{1}\right)$.

Suppose that $\varphi(f)(x)$ does not depend explicitly on $x$. Then

$$
\varphi(f)(x)=G\left(f(x), f^{\prime}\left(z_{1}\right)\right)
$$

for some rational function $G: R^{2} \rightarrow R$. Since $\varphi(f)\left(\alpha_{f}\right)=\alpha_{f}$,

$$
G\left(0, f^{\prime}\left(\alpha_{f}\right)\right)=\alpha_{f}
$$

for all $f \in D$. Clearly, for any real numbers $s, t(t \neq 0)$, there exists $f \in D$ such that

$$
\alpha_{f}=s, \quad f^{\prime}\left(\alpha_{f}\right)=t .
$$

By (5.1) we have

$$
G(0, t)=s
$$


for all $(t, s)$ with $t \neq 0$. This is a contradiction.

LEMMA 5.2. If $\varphi \in \Omega_{2}, p(\varphi) \geqq 2$, and $\varphi$ uses evaluations of $f$ only, then $a(\varphi) \geqq 5$.

Proof. We assume notation used in Case 1 of the proof of Theorem 4.4. Suppose $\mu_{n} \neq 0$ and $\mu_{i}=0$ for $i<n$. From (4.19) we can easily see that $n>0$.

Case 1. $(n=1)$. Suppose $a_{1}=0$. Then $\mu_{1}=c_{1,0}+c_{0,1}$. But $-f^{\prime} \nu_{2}=\mu_{1} \neq 0$, a contradiction. Hence $a_{1} \neq 0$.

By (4.23),

$$
\begin{aligned}
& c_{1,0}+c_{0,1}\left(1+a_{1} f^{\prime}\right) \neq 0, \\
& c_{1,0}=-c_{0,1}, \\
& b_{1,1}=b_{0,2}=0, \\
& b_{2,0}=-a_{1} c_{0,1} .
\end{aligned}
$$

Hence

$$
\varphi(f)(x)=x-\frac{a_{1} f^{2}(x)+\cdots}{f\left(z_{1}\right)-f(x)+\cdots} .
$$

Since the higher order terms in $f(x)$ cannot cancel the terms shown, the theorem is proved for this case.

Case 2. ( $n \geqq 2$ and $\left.a_{1} \neq 0\right)$. By (4.23) we know that some $b_{i, j} \neq 0$ for $i+j$ $=n+1 \geqq 3$. To compute $b_{i, j} f^{i} f^{j}\left(z_{1}\right)$ from $f$ and $f\left(z_{1}\right)$ needs at least 2 multiplications. The computation for getting $z_{1}$, the division and the combination with $x$ require another three arithmetic operations. Hence $a(\varphi) \geqq 5$.

Case 3. $\left(n \geqq 2, a_{1}=0\right)$. We can assume the notation used in Case 2 of the proof of Theorem 4.4. Since $z_{1}=x+a_{2} f^{2}+a_{3} f^{3}+\cdots$, to compute $z_{1}$ from $x$ and $f(x)$ requires at least two arithmetic operations. The division takes one arithmetic operation and to combine with $x$ requires another arithmetic operation. Hence it suffices to show that to compute $\sum_{0}^{q} c_{i, j} f^{i}(x) f^{j}\left(z_{1}\right)$ from $f(x)$ and $f\left(z_{1}\right)$ takes at least one arithmetic operation.

Suppose that $c_{1,0} \neq 0$. Then since by (4.32), $c_{1,0}+c_{0,1}=0, c_{0,1} \neq 0$. Hence to compute $\sum_{0}^{q} c_{i, j} f^{i}(x) f^{j}\left(z_{1}\right)$ from $f\left(z_{1}\right)$ needs at least one arithmetic operation. Suppose, on the other hand, that $c_{1,0}=0$. Then $c_{0,1}=0$. Since $\mu_{n} \neq 0$, by (4.3), $c_{i, j} \neq 0$ for some $i, j$ with $i+j \geqq 2$. Hence to compute $\sum_{0}^{q} c_{i, j} f^{i}(x) f^{j}\left(z_{1}\right)$ from $f(x)$ and $f\left(z_{1}\right)$ again needs at least one arithmetic operation.

For any $f \in D$, define

$$
E_{2}(f)=\sup _{\varphi \in \Omega_{2}} e(\varphi, f)
$$

Then $E_{2}(f)$ is the optimal efficiency achievable by a rational two-evaluation iteration without memory with respect to $f$. By Theorem 4.4 and Lemmas 5.1 and 5.2 , we have

$$
E_{2}(f) \leqq \max \left(\frac{1}{c(f)+c\left(f^{\prime}\right)+2}, \frac{1}{2 c(f)+5}\right)
$$


Consider Newton iteration $\gamma$ and the iteration $\psi$ defined by (1.2) and (1.3), respectively. We have

$$
e(\gamma, f)=\frac{1}{c(f)+c\left(f^{\prime}\right)+2}
$$

and

$$
e(\psi, f)=\frac{1}{2 c(f)+5}
$$

From (5.2), (5.3) and (5.4) we have the main result on optimal efficiency.

THEOREM 5.1 .

(i) If $c\left(f^{\prime}\right) \leqq c(f)+3$, then

$$
E_{2}(f)=\frac{1}{c(f)+c\left(f^{\prime}\right)+2}
$$

i.e., Newton iteration is optimal.

(ii) If $c\left(f^{\prime}\right) \geqq c(f)+3$, then

$$
E_{2}(f)=\frac{1}{2 c(f)+5}
$$

i.e., the iteration $\psi$ defined by (1.4) is optimal.

\section{REFERENCES}

[1] R. BRENT, S. WINOGRAD AND P. WOLFE, Optimal iterative processes for root-finding, Numer. Math., 20 (1973), pp. 327-341.

[2] A. C. HindmARsh, Optimality in a class of rootfinding algorithms, this Journal, 9 (1972), pp. 205-214.

[3] H. T. Kung And J. F. Traub, Optimal order of one-point and multipoint iteration, J. Assoc. Comput. Mach., 21 (1974), pp. 643-651.

[4] - Computational complexity of one-point and multipoint iteration, Complexity of Computation, R. M. Karp, ed., SIAM-AMS Proc., vol. 7, American Mathematical Society, Providence, R.I., 1974, pp. 149-160.

[5] J. Rissanen, On optimum root-finding algorithms, J. Math. Anal. Appl., 36 (1971), pp. 220-225.

[6] I. F. STEFFENSEN, Remarks on iteration, Skand. Aktuarietidskr., 16 (1933), pp. 64-72.

[7] J. F. Traub, On functional iteration and calculation of roots, Proc. 16th National ACM Conference, 1961.

[8] - Iterative Methods for the Solution of Equations, Prentice-Hall, Englewood Cliffs, N.J., 1964.

[9] — Computational complexity of iterative processes, SIAM J. Comput., 1 (1972), pp. 167-179.

[10] - Theory of optimal algorithms, Software for Numerical Mathematics, D. J. Evans, ed., Academic Press, New York, 1974, pp. 1-13.

[11] _ An introduction to some current research in numerical computational complexity, The Influence of Computing on Mathematical Research and Education, J. P. Lasalle, ed., American Mathematical Society, Providence, R.I., 1974, pp. 47-55.

[12] H. WOZNIAKOWSKI, Maximal stationary iterative methods for the solution of operator equations, this Journal, 11 (1974), pp. 934-949. 